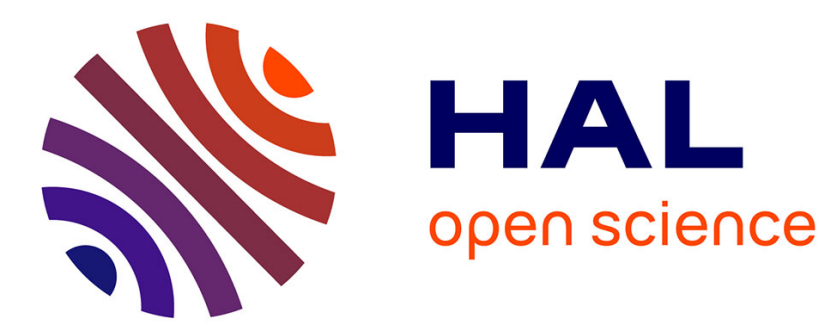

\title{
Non Data-Aided Estimation of Time-Varying Multiscale Doppler in Underwater Acoustic Channels
}

\author{
François-Xavier Socheleau
}

\section{To cite this version:}

François-Xavier Socheleau. Non Data-Aided Estimation of Time-Varying Multiscale Doppler in Underwater Acoustic Channels. Underwater Communications and Networking Conference (Ucomms), Aug 2021, Lerici, Italy. 10.1109/UComms50339.2021.9598120 . hal-02904488

\section{HAL Id: hal-02904488 \\ https://imt-atlantique.hal.science/hal-02904488}

Submitted on 24 Feb 2022

HAL is a multi-disciplinary open access archive for the deposit and dissemination of scientific research documents, whether they are published or not. The documents may come from teaching and research institutions in France or abroad, or from public or private research centers.
L'archive ouverte pluridisciplinaire HAL, est destinée au dépôt et à la diffusion de documents scientifiques de niveau recherche, publiés ou non, émanant des établissements d'enseignement et de recherche français ou étrangers, des laboratoires publics ou privés. 


\title{
Non Data-Aided Estimation of Time-Varying Multiscale Doppler in Underwater Acoustic Channels
}

\author{
Francois-Xavier Socheleau \\ IMT-Atlantique, Lab-STICC, fx.socheleau@imt-atlantique.fr
}

\begin{abstract}
In the context of underwater acoustic communications, we propose a non data-aided Doppler estimation method dedicated to single-carrier linearly-modulated signals. This method relies on the framework of time-warped cyclostationary random processes. It can estimate time-varying Doppler scales resulting from acceleration of up to several meters per second squared. It can also estimate multiple Doppler scales as observed in multiscale-multilag channels.
\end{abstract}

\section{INTRODUCTION}

In the underwater acoustic channel, the motion-induced Doppler effect either compresses or dilates signals in time. This results from the wideband characteristic of the channel combined with the low speed of sound. Such a Doppler scaling must be estimated and compensated at reception so as to correctly retrieve the transmitted information.

Doppler scale estimation has been widely addressed in the literature and various models have been considered. For instance, in [1]-[4], the Doppler scale is common between channel paths and constant (or slowly varying) over a data packet. [5]-[7] assume multipath signals with different Doppler scales but constant (or slowly varying) in time. Finally, [8] considers multipath signals with a common but fast-varying Doppler scale. All these methods either require a significant pilot overhead or are limited in their capability of tracking (multiple) fast-varying Doppler scales.

In this work, we propose an estimator capable of estimating time-varying Doppler scales resulting from strong accelerations with reasonable complexity. This estimator is dedicated to single-carrier linearly-modulated signals and can estimate multiple Doppler scales such as those observed in multiscalemultilag channels [9]. In addition, it is non data-aided so as not to increase overheads and to be applicable to noncooperative contexts. As opposed to existing non data-aided estimators, such as [3] and [8], our approach does not rely on some correlation induced by a very specific data framing. The proposed estimator takes advantage of the cyclostationary features of PSK/QAM signals that become time-warped as a result of Doppler scaling [10]. More specifically, a quadratic function of time is used to model the motion-induced Doppler and a cost function is derived based on the cyclostationary features. This cost is then optimized to estimate the polynomial coefficients related to speed and acceleration.

The rest of the paper is organized as follows. The signal model and the assumptions are formulated in Sec. II. Sec.
III presents the cyclostationary features of linearly-modulated signals as well as the single Doppler scale estimator. This estimator is extended to multipath signals with different Doppler scales in Sec. IV. Numerical results are provided in Sec. V, followed by conclusions in Sec. VI.

\section{PROBlem Formulation}

We consider a baseband multiscale-multilag channel model that maps an input signal $x(t)$ into an output signal $r(t)$ according to the following relationship ${ }^{1}$

$$
r(t)=\sum_{\ell=1}^{L} \lambda_{\ell}(t) y_{\ell}(t)+w(t),
$$

where $\lambda_{\ell}(t)$ is the complex attenuation of the $\ell$-th channel tap, $w(t)$ is the additive noise assumed to be wide-sense stationary and $y_{\ell}(t)$ is a delayed, phase shifted and time-warped version of the transmitted signal, i.e.,

$$
y_{\ell}(t)=x\left(t-\tau_{\ell}-\phi_{\ell}(t)\right) e^{-i 2 \pi f_{c}\left(\tau_{\ell}+\phi_{\ell}(t)\right)} .
$$

$\tau_{\ell}$ denotes the initial time of arrival of the $\ell$-th path, $f_{c}$ is the carrier frequency and $\phi_{\ell}(t)$ is the time-warping function resulting from Doppler scaling. $x(t)$ is assumed to be a PSK or QAM signal expressed as

$$
x(t)=\sum_{k \in \mathbb{Z}} b_{k} g\left(t-k T_{s}\right),
$$

where $b_{k}$ denotes the transmitted symbols, $g(\cdot)$ is the pulseshaping filter and $T_{s}$ is the symbol duration assumed to be known by the receiver. The symbols $b_{k}$ are assumed to be zeromean identically and independently distributed. Each function $\phi_{\ell}(t)$ is usually modeled as the sum of a dominant term, compensated by resampling at reception, plus some residual Doppler shift that can be tracked with a PLL-like approach. We here focus on the dominant term and model the relative motion between the transmitter (TX) and the receiver (RX) with the following polynomial $[8, \mathrm{Eq} .(9)]$

$$
\phi_{\ell}(t)=\frac{v_{\ell}}{c} t+\frac{a_{\ell}}{2 c} t^{2}
$$

where $c$ is the sound speed, $v_{\ell}$ is the relative speed between $\mathrm{TX}$ and RX, and $a_{\ell}$ is the relative acceleration. Given the observation $r(t)$, the aim of this work is to estimate $\phi_{\ell}(t)$ (or, equivalently, $v_{\ell}$ and $a_{\ell}$ ) without the knowledge of the symbols $b_{k}$.

${ }^{1}$ For the sake of simplicity, the frequency-dependent losses are voluntarily omitted in the channel model. 


\section{CYClOSTATIONARY-BASED ESTIMATION OF A SINGLE DOPPLER SCALE}

PSK and QAM signals are known to be cyclostationary [11]. More specifically, let $R_{x}(t, u)$ be the autocorrelation function defined as

$$
R_{x}(t, u)=\mathbb{E}\left[x^{*}(t) x(t+u)\right] .
$$

If $x(t)$ is second-order cyclostationary, $R_{x}(t, u)$ admits a Fourier series expansion such that

$$
R_{x}(t, u)=\sum_{\alpha \in \mathcal{A}} R_{x}^{\alpha}(u) e^{i 2 \pi \alpha t}
$$

where $R_{x}^{\alpha}(u)$ is the cyclic-autocorrelation function defined as

$$
R_{x}^{\alpha}(u)=\lim _{T \rightarrow \infty} \frac{1}{T} \int_{-\frac{T}{2}}^{\frac{T}{2}} R_{x}(t, u) e^{-i 2 \pi \alpha t} \mathrm{~d} t .
$$

$\mathcal{A}$ denotes the countable set of cycle frequencies $\alpha$ that are integer multiples of the reciprocal of some period. In the particular case of PSK/QAM signals, it can be shown that the autocorrelation function is $T_{s}$-periodic when $u=0$ so that [11, Sec. 5.2]

$$
R_{x}(t, 0)=\sum_{n \in \mathbb{Z}} R_{x}^{\frac{n}{T_{s}}}(0) e^{i 2 \pi \frac{n}{T_{s}} t}
$$

where

$$
R_{x}^{\frac{n}{T_{s}}}(0)=\frac{\mathbb{E}\left[\left|b_{k}\right|^{2}\right]}{T_{s}} \int_{\mathbb{R}}|g(t)|^{2} e^{-i 2 \pi \frac{n}{T_{s}} t} \mathrm{~d} t .
$$

For real-valued pulse shaping functions $g$ of Fourier transform $G$, the cyclic-autocorrelation function also satisfies

$$
R_{x}^{\frac{n}{T_{s}}}(0)=\frac{\mathbb{E}\left[\left|b_{k}\right|^{2}\right]}{T_{s}}(G \circledast G)\left(\frac{n}{T_{s}}\right),
$$

where $\circledast$ denotes convolution. For most pulse shaping functions used in practice, the magnitude of $(G \circledast G)\left(\frac{n}{T_{s}}\right)$ drops very quickly with $n$ or is even null for $|n|>1$. Therefore, $n$ is usually restricted to the set $\{-1,0,1\}$ in Eq. (8).

Although $x(t)$ is cylostationary, Doppler scaling expressed in (2) destroys the cyclic feature of the received signal, i.e., $R_{y_{\ell}}(t, 0)$ is not periodic. Such a process is known as a timewarped cyclostationary process [10]. However, if the timewarping functions $\phi_{\ell}(t)$ are known, the original cyclic feature can be restored. This is expressed by the following relationship

$$
R_{x}^{\frac{n}{T_{s}}}(0)=\lim _{T \rightarrow \infty} \frac{e^{i 2 \pi \frac{n}{T_{s}} \tau_{\ell}}}{T} \int_{-\frac{T}{2}}^{\frac{T}{2}} R_{y_{\ell}}(t, 0) e^{-i 2 \pi \frac{n}{T_{s}}\left(t-\phi_{\ell}(t)\right)} \mathrm{d} t .
$$

This fact can be used to estimate the Doppler scale. Assume for now that the channel exhibits a single Doppler scale, i.e., $\phi_{\ell}(t)=\phi(t), \forall \ell$. From Eqs. (1), (8), (10) and (11) it can be shown that

$$
\phi(t)=\underset{\varphi(t)}{\operatorname{argmax}}\left|\lim _{T \rightarrow \infty} \frac{1}{T} \int_{-\frac{T}{2}}^{\frac{T}{2}} R_{r}(t, 0) e^{-i \frac{2 \pi}{T_{s}}(t-\varphi(t))} \mathrm{d} t\right|^{2} .
$$

In practice, we cannot observe $R_{r}(t, 0)=\mathbb{E}\left[|r(t)|^{2}\right]$ so that it is replaced by the simple unbiased time-varying estimate $|r(t)|^{2}$. Moreover, since Doppler scaling is due to relative

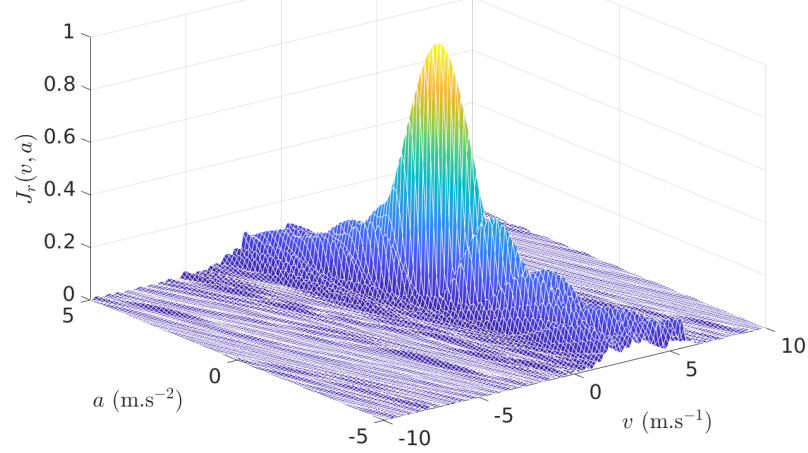

Fig. 1. Example of a cost function $J_{r}$ obtained with a QPSK signals propagated in a shallow water channel using a time-varying simulation with Bellhop [12]. $(\widehat{v}, \widehat{a})=(1,0.5) . T_{o b s}=1 \mathrm{~s}, T_{s}=0.2 \mathrm{~ms}, f_{c}=20 \mathrm{kHz}$, $E_{s} / N_{0}=10 \mathrm{~dB}$

motion, a polynomial model such as the one given in (4) can be used. Over some time interval $T_{\text {obs }}, \phi(t)$ can therefore be estimated as

$$
\widehat{\phi}(t)=\frac{\widehat{v}}{c} t+\frac{\widehat{a}}{2 c} t^{2} \text { with }(\widehat{v}, \widehat{a})=\underset{(v, a)}{\operatorname{argmax}} J_{r}(a, v)
$$

and

$$
J_{r}(a, v)=\left.\left.\frac{1}{T_{\text {obs }}}\left|\int_{0}^{T_{\mathrm{obs}}}\right| r(t)\right|^{2} e^{-i \frac{2 \pi}{T_{s}}\left(t-\frac{v}{c} t-\frac{a}{2 c} t^{2}\right)} \mathrm{d} t\right|^{2} .
$$

Eqs. (12) and (13) exploit the fact that Doppler scaling changes the periodic function $R_{x}(t, 0)$ into a chirp function $R_{r}(t, 0)$. An example of the cost function $J_{r}$ is shown in Fig. 1. Given the shape of this function, the optimization can be done by first performing a coarse-grid search and by then applying a gradient approach.

\section{ESTIMATION OF MULTIPLE DOPPLER SCALES}

In the case where the channel is multiscaled, $R_{r}(t, 0)$ can be expressed as the sum of several chirp signals whose timevarying phases must be estimated. More precisely,

$$
\begin{aligned}
R_{r}(t, 0)= & \sum_{\ell=1}^{L} \sum_{m=1}^{L} \mathbb{E}\left[\lambda_{\ell}(t) \lambda_{m}^{*}(t)\right] \mathbb{E}\left[y_{\ell}(t) y_{m}^{*}(t)\right] \\
& +\mathbb{E}\left[|w(t)|^{2}\right] \\
\stackrel{(a)}{\approx} & \sum_{\ell=1}^{L} \mathbb{E}\left[\left|\lambda_{\ell}(t)\right|^{2}\right] \mathbb{E}\left[\left|y_{\ell}(t)\right|^{2}\right]+\mathbb{E}\left[|w(t)|^{2}\right] \\
\stackrel{(b)}{=} & \sum_{\ell=1}^{L} \mathbb{E}\left[\left|\lambda_{\ell}(t)\right|^{2}\right] \sum_{n=-1}^{1} R_{x}^{\frac{n}{T_{s}}}(0) e^{i 2 \pi \frac{n}{T_{s}}\left(t-\tau_{\ell}-\phi_{\ell}(t)\right)} \\
& +\mathbb{E}\left[|w(t)|^{2}\right]
\end{aligned}
$$

where (a) follows from the assumption that $\left|\tau_{m}-\tau_{\ell}\right|>T_{s}$ for most $m \neq \ell$ and (b) follows from (11). Approximation (a) is a way of stating that paths with significantly different Doppler 
scales should be resolved within the signal bandwidth. If not, an interfering term appears in the autocorrelation function and make it difficult to differentiate the contribution of each timewarping function. Since the DC component of $R_{r}(t, 0)$ does not carry any information on $\phi_{\ell}(t)$, it can be subtracted in the previous expression so that

$$
\begin{aligned}
& R_{r}(t, 0)-R_{r}^{0}(0)= \\
& \quad \sum_{\ell=1}^{L} \mathbb{E}\left[\left|\lambda_{\ell}(t)\right|^{2}\right] 2 \Re\left\{R_{x}^{\frac{1}{T_{s}}}(0) e^{i \frac{2 \pi}{T_{s}}\left(t-\tau_{\ell}-\phi_{\ell}(t)\right)}\right\} .
\end{aligned}
$$

For tractability, we assume that $\lambda_{\ell}(t)$ is wide-sense stationary on the observation time interval, i.e., $\mathbb{E}\left[\left|\lambda_{\ell}(t)\right|^{2}\right]=\mathbb{E}\left[\left|\lambda_{\ell}\right|^{2}\right]$, $\forall t \in\left[0, T_{\text {obs }}\right]$. In addition, because of the transmission geometry, it is very common in practice that several paths share (nearly) the same time-warping function $\phi_{\ell}$. Let $\mathcal{L}_{d}$ denote the set of path indexes with the same function $\phi_{d}$. Assuming that the channel contains $D \leq L$ different Doppler scales, a more practical formulation of (15) may then be

$$
R_{r}(t, 0)-R_{t}^{0}(0)=\sum_{d=1}^{D} 2 \Re\left\{\Gamma_{d} e^{i \frac{2 \pi}{T_{s}}\left(t-\phi_{d}(t)\right)}\right\},
$$

where $\Gamma_{d}=\sum_{\ell \in \mathcal{L}_{d}} \mathbb{E}\left[\left|\lambda_{\ell}\right|^{2}\right] R_{x}^{\frac{1}{T_{s}}}(0) e^{-i \frac{2 \pi}{T_{s}} \tau_{\ell}}$. Let $\operatorname{card}(\cdot)$ denote the cardinality of a set, then we have $\sum_{d=1}^{D} \operatorname{card}\left(\mathcal{L}_{d}\right)=$ $L$.

The proposed estimation procedure relies on (16) and is iterative. At each iteration, the dominant Doppler scale can be estimated using (13). Then, the contribution of the dominant "Doppler chirp" in (16) can be subtracted after estimating its amplitude $\Gamma$. The procedure is repeated until a stopping criterion is reached. Similarly to the approach described in Sec. III, we cannot observe $R_{r}(t, 0)=\mathbb{E}\left[|r(t)|^{2}\right]$ so that it is replaced by $|r(t)|^{2}$ in (16). The full method is detailed in Algorithm 1. There can be several ways of stopping the iterative procedure. It can be stopped once a given number $D_{\max }$ of significant Doppler scales is reached. This number could be (roughly) known a priori based on the knowledge of the transmission geometry. Another approach could be to stop the procedure once the combined amplitudes of the paths with the same Doppler scale is considered to be too small to be of interest, i.e., when $\left|\widehat{\Gamma}^{(n)}\right| /\left|\widehat{\Gamma}^{(0)}\right|<\epsilon$ in Algo. 1 .

\section{Numerical Results}

This section presents initial results obtained from numerical simulations with Bellhop [12]. In a first scenario, a multilag single-scale channel is considered. The performance of the proposed method is assessed and compared with the state-ofthe-art method detailed in [8]. Our method is then tested when faced to a multiscale-multilag channel.

\section{A. Single Doppler scale estimation}

In this scenario, we consider a shallow water (SW) channel with a constant sound speed profile. The water, TX and RX depths are set to 50,10 and $35 \mathrm{~m}$, respectively. The initial transmit range is set to $2 \mathrm{~km}$. Root-raised cosine filtered

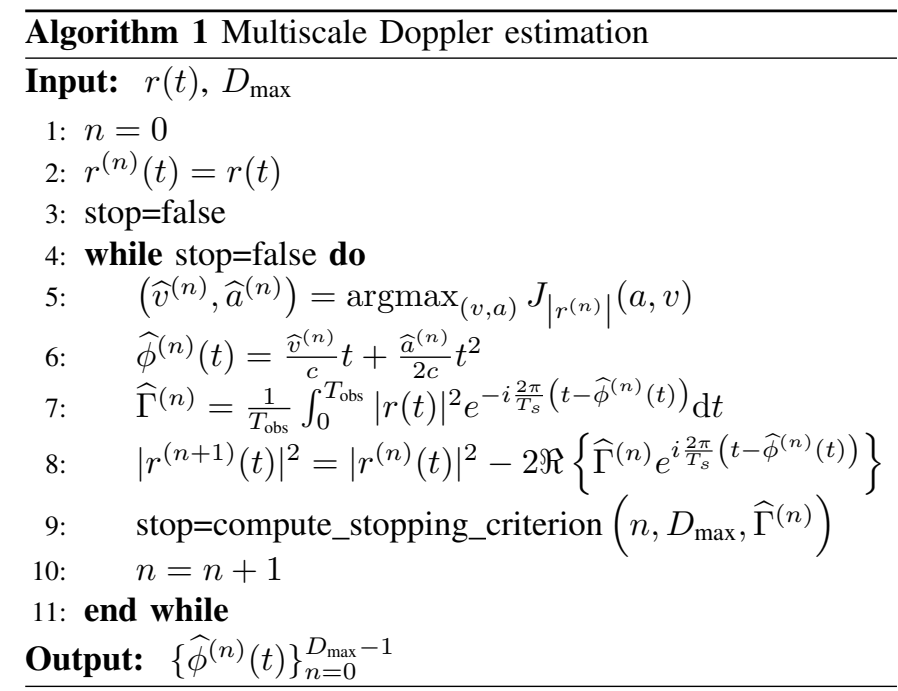

QPSK signals are simulated with $T_{o b s}=1 \mathrm{~s}, T_{s}=0.2$ $\mathrm{ms}, f_{c}=20 \mathrm{kHz}$, a roll-off set to 0.5 and a (baseband) sampling rate of $20 \mathrm{kHz}$. The TX moves horizontally with a radial speed and acceleration randomly drawn from the set $\left[-v_{m}, v_{m}\right] \times\left[-a_{m}, a_{m}\right]$, with $v_{m}=10 \mathrm{~m} . \mathrm{s}^{-1}$ and $a_{m}=1$ $\mathrm{m} . \mathrm{s}^{-2}$. Bellhop is used to model the time-varying multipath distortions due to relative motion. 100 Bellhop impulse responses per second are generated along the TX/RX trajectory. These impulse responses are then linearly interpolated in time to match the sampling frequency of the transmitted signal. Our method is compared with [8]. This method, known as the "Multibranch Autocorrelation" (MBA) is able to estimate the speed as well as the acceleration for systems using periodic pilot signals or repetitive data transmission. We consider the latter configuration which is semi-blind. In this case, the receiver does not know the value of the data symbols but exploits the redundancy between consecutive data blocks. As opposed to our approach, this method reduces the useful data rate by 2 . We use the root mean squared error (RMSE) of the estimate $\widehat{\phi}(t)$ as our performance metric. It is defined as

$$
\mathrm{RMSE}=\sqrt{\frac{\mathbb{E}\left[\int_{0}^{T_{o b s}}(\hat{\phi}(t)-\phi(t))^{2} \mathrm{~d} t\right]}{\mathbb{E}\left[\int_{0}^{T_{o b s}} \phi^{2}(t) \mathrm{d} t\right]}} .
$$

Fig. 2 shows the estimated RMSE for both methods as a function of $E_{s} / N_{0}$, where $E_{s}$ is the signal energy per information symbol and $N_{0}$ is the power spectral density of the additive white Gaussian noise. RMSE is estimated with 300 Monte-Carlo trials per $E_{s} / N_{0}$. As a reference, the RMSE of the dummy estimator $\widehat{\phi}(t)=t$ is also plotted. For comparison, the performance obtained in an AWN channel with Doppler scaling is shown as well. The parameters of method [8] are set as explained in [13, Sec. IV]: delay step $=50 \mu$ s and frequency step $=1 \mathrm{~Hz}$. The solution of (13) is found by first applying a coarse grid search with a step of $1 / 6 \mathrm{~m} . \mathrm{s}^{-1}$ for speed and 1/2 $\mathrm{m} . \mathrm{s}^{-2}$ for acceleration. A Barzilai Borwein gradient approach is then applied to get more precise results [14].

The performance curves exhibit the three typical regions observed in non-linear estimation [15], [16]: the no informa- 


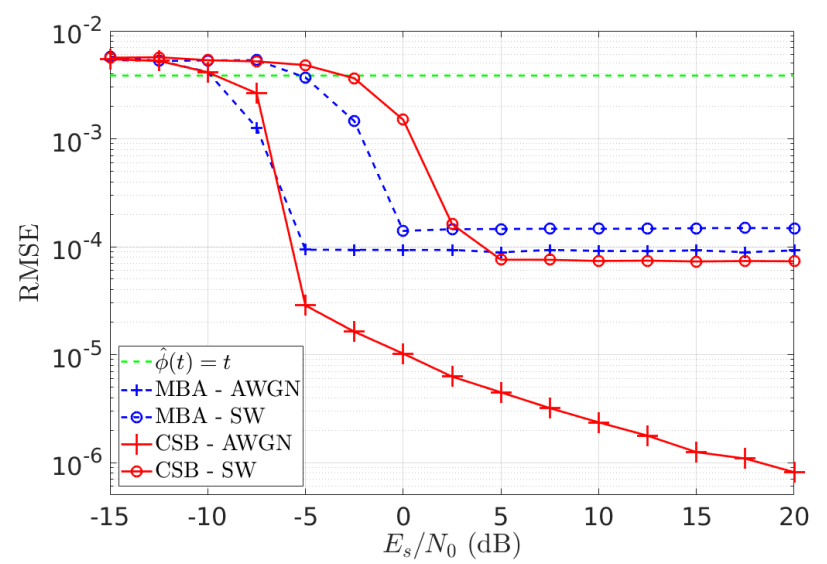

Fig. 2. Performance comparison between the multibranch autocorrelation (MBA) estimator and the cyclostationary-based (CSB) method.

tion region driven by ambiguity errors at very low $E_{s} / N_{0}$, the threshold region that characterizes the transition from global to local errors and the asymptotic region at high $E_{s} / N_{0}$ driven by mainlobe errors. For both channels, our approach (CSB) gives better performance in the asymptotic region. MBA is more efficient in the threshold region but this gain costs half the data rate. MBA shows RMSE flooring caused by grid search optimization with finite grid step. For our method in the SW channel, RMSE flooring is also observed in the asymptotic region. This is due to the very rich multipath environment and residual Doppler scaling differences between taps.

Both methods can be implemented using FFTs and have therefore order of $\mathcal{O}(N \log (N))$ complexity, where $N$ is the number of signal samples over the duration $T_{\text {obs }}$. However, the actual complexity depends on constant factors that may be slightly different between the two methods. For instance, in this specific example, MBA is twice more complex than CSB because the grid step used for the cost function optimization is smaller than the one used in our method. In addition, as discussed in [8, Sec. III-B], if higher accelerations were to be considered, MBA would require signal resampling that would result in a significant complexity burden. This is not the case for CSB as its complexity increases linearly with $a_{m}$. Finally, as illustrated in Table I, CSB is robust to strong accelerations.

TABLE I

Performance of the CSB method as a function of the maximum acceleration $a_{m}$. AWGN channel with Doppler scaling, $v_{m}=10 \mathrm{~m} \cdot \mathrm{s}^{-1}, \frac{E_{s}}{N_{0}}=10 \mathrm{~dB}$.

\begin{tabular}{|c|cccc|}
\hline$a_{m}\left(\mathrm{~m}_{\mathrm{s}}{ }^{-2}\right)$ & $\mathbf{0 . 5}$ & $\mathbf{1}$ & $\mathbf{2}$ & $\mathbf{4}$ \\
\hline$R M S E\left(\times 10^{-6}\right)$ & 2.35 & 2.34 & 2.55 & 2.51 \\
\hline
\end{tabular}

\section{B. Multiple Doppler scale estimation}

We now consider a deep-water multiscale-multilag channel with a Munk sound speed profile [12]. All the simulation parameters are identical to those described in the previous section, except that the water and RX depths are set to 5000 and $200 \mathrm{~m}$, respectively. The initial TX depth is 25 $\mathrm{m}$, the TX/RX range is set to $200 \mathrm{~m}$ and the transmitter

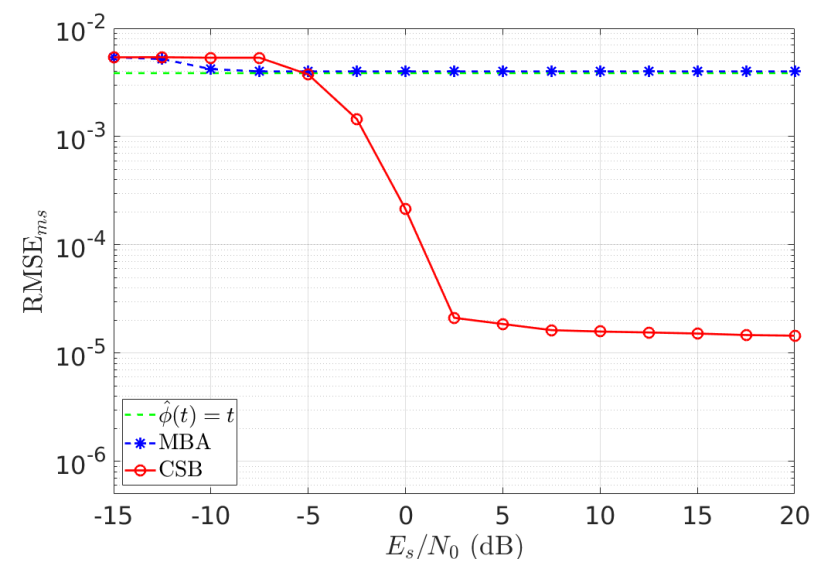

Fig. 3. Performance of the cyclostationary-based (CSB) method in a multiscale-multilag channel.

moves towards the surface. Such a configuration is known to be difficult for communications systems as the time-varying channel is mostly made of two paths with similar amplitudes and opposite-sign Doppler scales. For multiscale channels, the performance metric is now defined as:

$$
\mathrm{RMSE}_{\mathrm{ms}}=\sqrt{\frac{\sum_{d=1}^{D_{\max }}\left|\Gamma_{d}\right| \times \mathbb{E}\left[\int_{0}^{T_{o b s}}\left(\widehat{\phi}_{d}(t)-\phi_{d}(t)\right)^{2} \mathrm{~d} t\right]}{\sum_{d=1}^{D_{\max }}\left|\Gamma_{d}\right| \times \mathbb{E}\left[\int_{0}^{T_{o b s}} \phi_{d}^{2}(t) \mathrm{d} t\right]}},
$$

where $D_{\max }=2$ in this specific scenario.

The MBA curve in Fig. 3 shows that using a single Doppler scale estimator in a multiscale environment can be worse than not performing any estimation at all (i.e, $\widehat{\phi}(t)=t$ ). It also shows that CSB can be highly efficient in multiscale channels. In our scenario, the asymptotic regime is reached for a value of $E_{s} / N_{0}$ as low as $2.5 \mathrm{~dB}$. Detecting and estimating multiple Doppler scales can be useful to trigger post-processing to make the communication link more robust. For instance, it can be used to optimize the choice of the equalizer or PLL parameters. It can also trigger a change of waveform if the transmitter is adaptive, or some array-processing to separate multipath signals with different scales.

\section{CONCLUSION}

Based on the framework of time-warped cyclostationary signals, we have proposed an estimator of time-varying Doppler scales for linearly-modulated signals. The estimator is built from the autocorrelation function of the received signal. We have shown that this function can be expressed as the sum of several chirp signals whose time-varying phases are Dopplerdependent. Numerical simulations indicate that the method provides very good performance in difficult multilag, and possibly multiscale, environments. We think that the performance could be further improved by considering non-zero lags in Eq. (8). Although dedicated to PSK or QAM signals, the proposed framework could also be extended to other waveforms exhibiting cyclostationarity such as OFDM signals with a cyclic-prefix. 


\section{REFERENCES}

[1] B.S. Sharif, J. Neasham, O.R. Hinton, and A.E. Adams, "A Computationally Efficient Doppler Compensation System for Underwater Acoustic Communications," IEEE J. Ocean. Eng., vol. 25, no. 1, 2000.

[2] S. F. Mason, C. R. Berger, S. Zhou, and P. Willett, "Detection, synchronization, and doppler scale estimation with multicarrier waveforms in underwater acoustic communication," IEEE Journal on selected areas in communications, vol. 26, no. 9, pp. 1638-1649, 2008.

[3] P. Cotae and TC Yang, "A cyclostationary blind doppler estimation method for underwater acoustic communications using direct-sequence spread spectrum signals," in 2010 8th International Conference on Communications. IEEE, 2010, pp. 323-326.

[4] S. Imbert, G. Ansel, and C. Laot, "Frame detection and synchronization for a simo receiver in presence of doppler shift for underwater acoustic communications," in OCEANS 2019-Marseille. IEEE, 2019, pp. 1-4.

[5] T. J. Riedl and A. C. Singer, "Broadband doppler compensation: Principles and new results," in 2011 Conference Record of the Forty Fifth Asilomar Conference on Signals, Systems and Computers (ASILOMAR). IEEE, 2011, pp. 944-946.

[6] Y. Zhao, H. Yu, G. Wei, F. Ji, and F. Chen, "Parameter estimation of wideband underwater acoustic multipath channels based on fractional fourier transform," IEEE Transactions on Signal Processing, vol. 64, no. 20 , pp. 5396-5408, 2016.

[7] S. Yerramalli and U. Mitra, "Optimal resampling of ofdm signals for multiscale-multilag underwater acoustic channels," IEEE Journal of Oceanic Engineering, vol. 36, no. 1, pp. 126-138, 2011.
[8] J. Li, Y. V. Zakharov, and B. Henson, "Multibranch autocorrelation method for doppler estimation in underwater acoustic channels," IEEE Journal of Oceanic Engineering, vol. 43, no. 4, pp. 1099-1113, 2017.

[9] P. A. van Walree and R. Otnes, "Ultrawideband Underwater Acoustic Communication Channels," IEEE J. Ocean. Eng., vol. 38, no. 4, pp. 678-688, October 2013.

[10] A. Napolitano, "Time-warped almost-cyclostationary signals: characterization and statistical function measurements," IEEE Transactions on Signal Processing, vol. 65, no. 20, pp. 5526-5541, 2017.

[11] W. A. Gardner, A. Napolitano, and L. Paura, "Cyclostationarity: Half a century of research," Signal processing, vol. 86, no. 4, pp. 639-697, 2006.

[12] M. B. Porter, "The bellhop manual and user's guide: Preliminary draft," Heat, Light, and Sound Research, Inc., La Jolla, CA, USA, Tech. Rep, 2011.

[13] Y. Zakharov, B. Henson, R. Diamant, Y. Fei, P. D. Mitchell, N. Morozs, L. Shen, and T. C. Tozer, "Data packet structure and modem design for dynamic underwater acoustic channels," IEEE Journal of Oceanic Engineering, vol. 44, no. 4, pp. 837-849, 2019.

[14] J. Barzilai and J. M. Borwein, "Two-point step size gradient methods," IMA journal of numerical analysis, vol. 8, no. 1, pp. 141-148, 1988.

[15] H.L. Van Trees, Detection, Estimation, and Modulation Theory Part I, Wiley, 1968.

[16] Y. Le Gall, F.-X. Socheleau, and J. Bonnel, "Matched-field processing performance under the stochastic and deterministic signal models," IEEE Trans. Signal Process., vol. 62, no. 22, pp. 5825-5838, 2014. 